Land, damit sich unsere Beziehungen verbessern und sich auf der Grundlage guten gegenseitigen Vorteils entwickeln.

Übermitteln Sie Ihrem Premierminister Grüße und beste Wünsche. Ich habe von ihm einen Brief als Antwort auf den Brief erhalten, den wir ihm geschickt hatten. Er stellt einige interessante Fragen. Insgesamt sind wir über seinen Brief befriedigt und bereiten eine Antwort vor, um ihm unsere Haltung verständlich zu machen. Die Frage betrifft genau den Vorschlag der Errichtung einer Inspektion in der Arktis. Am Anfang unseres Gesprächs haben wir Ihnen den Standpunkt der Sowjetunion dazu dargelegt. Ich wünsche Ihnen gute Gesundheit. Auf Wiedersehen.

Das Gespräch dauerte 1 Stunde, 50 Minuten.

Übersetzung aus dem russischen Originaltext RGANI, fond 52, opis‘ 1 , delo 569, Bl. 49-72

\title{
25. Rede Chruschtschows auf der Beratung der Vertreter der kommunistischen und Arbeiterparteien der sozialistischen Länder in Moskau am 21. Mai 1958
}

Werte Genossen! Ich möchte die Ansicht der sowjetischen Delegation zum Ausdruck bringen zu der Frage, die in der Aufzeichnung des Gen. Ulbricht aufgeworfen wurde, die allen an dieser Beratung teilnehmenden Delegationen der kommunistischen und Arbeiterparteien zugegangen ist. ${ }^{457}$

Wir haben uns mit dieser Aufzeichnung bekannt gemacht und messen ihrem Inhalt eine sehr große Bedeutung bei. Würde man absehen von der kommunisti-

457 Die Aufzeichnung entsprach dem Inhalt des Schreibens, das vom Politbüro der SED am 13. Mai 1958 an Chruščëv gerichtet worden war. Darin hatte Ulbricht die sowjetische Führung um eine „Aussprache über einige Grundfragen“ gebeten, „die mit der Perspektive der Entwicklung in der DDR und in Deutschland zusammenhängen“. Es sei die Lage entstanden, dass einerseits die DDR sich seit Mitte 1957 weiter stabilisiert habe, andererseits jedoch von den NATO-Beschlüssen sowie der Propaganda und der Staatsmacht Westdeutschlands stärkerer Druck ausgehe. Die Adenauer-Regierung suche diesen mit der - kürzlich beschlossenen - „Atomrüstung“ weiter zu verstärken, um mit Mitteln bis hin zur „Provokation des Krieges“ die „Eingliederung“ der DDR zu erreichen, doch zeige die „Volksbewegung gegen den Atomtod“, „dass die Möglichkeit besteht, einen politischen Umschwung herbeizuführen“. Um „zu weiteren Erfolgen in der Friedensbewegung zu kommen“, sei es notwendig, dass die DDR vor Abschluss der westdeutschen Aufrüstung im Jahr 1961 nicht nur ihre Friedenspolitik fortführe, sondern „auch durch den Stand der Lebenshaltung und die demokratische und kulturelle Entwicklung für die Arbeiterklasse und die Werktätigen anziehend“ werde. Das Konzept, die DDR zum sozialistischen Schaufenster gegenüber dem Westen zu machen, habe jedoch „bisher nicht durchgeführt werden“ können. Angesichts der so dargestellten Situation wurden die UdSSR und die anderen sozialistischen Staaten um materielle Hilfe für die DDR gebeten; nur dadurch könne man die politischen Ziele in Deutschland erreichen. Chruščëv ließ sich von der Argumentation überzeugen und setzte sich für entsprechende Unterstützungsleistungen an die DDR ein. Dabei wandte er sich gegen die Ansicht, dass man in Ostdeutschland besser dran sei als anderswo. Das sei nicht das Kriterium, auf das es ankomme. Vielmehr gehe es um das übergeordnete Interesse des sozialistischen Lagers als Ganzem, in Deutschland als entscheidendem Land in der Mitte Europas den politischen Durchbruch zu erzielen. 
schen Weltsicht und der kommunistischen Solidarität, auf die jeder Kommunist verpflichtet ist, könnten wir natürlich die ernsten Fragen übergehen, die in dieser Aufzeichnung aufgeworfen werden. Doch wir Kommunisten können das nicht tun, weil es unserer internationalistischen Pflicht, unserem Verständnis der proletarischen Solidarität widerspricht. Uns der Prüfung und Regelung derartiger Fragen zu entziehen, ist für uns Kommunisten nicht möglich.

Es ist bekannt, dass die Völker der Sowjetunion, Polens, der Tschechoslowakei und anderer Länder in den letzten Jahrzehnten zweimal der Invasion der Deutschen ausgesetzt waren, und sie haben zwei grausige, verheerende Kriege durchgemacht, die von den deutschen Militaristen entfesselt worden waren. Wenn wir von bloß nationalen Erwägungen auszugehen hätten, fänden sich natürlich viele Gründe dafür, um uns den Fragen zu entziehen, die unsere deutschen Genossen aufgeworfen haben, und dafür eine Erklärung zu finden: Wir haben angeblich selbst den Hals voller Sorgen. Mehr noch, will man den jetzigen Lebensstandard Ostdeutschlands zugrunde legen, ist er natürlich nicht niedriger als in den anderen sozialistischen Ländern, an die unsere Genossen aus der Deutschen Demokratischen Republik sich jetzt wenden, und er kann sogar noch höher sein als bei vielen Staaten. Auch wir selbst schließen uns aus dieser Liste nicht aus; bei einer ganzen Reihe von Konsumgütern besetzt die DDR jetzt einen bedeutend höheren Platz als die Sowjetunion.

Wenn wir uns erinnern wollen an die gewaltigen Verheerungen, welche die Deutschen im letzten Krieg auf dem Territorium der Sowjetunion und anderer Länder angerichtet haben, und an die vielen Millionen von Menschenleben, die durch den Krieg jäh vernichtet worden sind, werden wir viele in sich schlüssige Gründe finden, die nicht nur unsere Ablehnung der Gewährung von Hilfe an die Deutschen rechtfertigen, sondern auch gerechten Zorn bei der Erinnerung an die Vergangenheit hervorrufen.

Aber wir Kommunisten haben auch die Pflicht, auf diese Dinge unter geschichtlichem Blickwinkel zu schauen. Wir müssen nach vorn blicken und unserer kommunistischen Bewegung den Weg frei machen, und deswegen sind wir dazu verpflichtet, von marxistisch-leninistischen Positionen aus an diese Fragen heranzugehen und die Vergangenheit so zu bewerten, dass sie uns nicht daran hindert, die vor uns stehenden Aufgaben richtig zu lösen, nicht daran hindert, unsere gemeinsame Bewegung voran, zum Kommunismus zu bringen.

Gestern hat der Vertreter der DDR in seiner Stellungnahme treffend gesagt, und auch in der Aufzeichnung steht es so, dass die DDR das Schaufenster der sozialistischen Länder zur kapitalistischen Welt ist. Die DDR ist, um es so auszudrücken, der Vorposten in unserem Kampf gegen den Kapitalismus. Dabei ist zu berücksichtigen, dass die DDR eine offene Grenze zur kapitalistischen Welt hat. 458 Das heutige Deutschland hat die Besonderheit, dass das deutsche Volk auf zwei Staaten verteilt ist, dass es in zwei Staaten lebt, die unterschiedliche Systeme haben. Und zwischen diesen Staaten verläuft keine geschlossene Grenze. Das

458 Die Aussage bezieht sich darauf, dass nach Schließung der Grenze zur Bundesrepublik Deutschland 1952 in Berlin weiterhin Freizügigkeit herrschte. Daher konnten sich Westbesucher ungehindert ein Bild von den Verhältnissen im Ostsektor machen. 
macht die Lage der DDR und unserer deutschen Genossen viel komplizierter. ${ }^{459}$ Darum müssen wir uns konkret und real in die Lage der kommunistischen DDRGenossen versetzen, bei denen ziemlich schwierige Umstände entstanden sind.

Genossen! Die Arbeiterklasse und die Werktätigen unseres Landes haben als erste die sozialistische Revolution, die Große Oktoberrevolution, durchgeführt. $\mathrm{Zu}$ dieser Zeit waren unsere Menschen halb verhungert, barfuß und halb nackt; wir hatten damals einen sehr niedrigen Lebensstandard. Wenn man eine Analogie anführen könnte, so würde ich sagen, dass man unsere damalige Lage mit der vergleichen könnte, die vor kurzem das chinesische Volk durchgemacht hat, das ebenfalls unter schweren Bedingungen die Revolution und einen Befreiungskampf durchgeführt, seine Wirtschaft wiederhergestellt und umfangreiche Maßnahmen zur Stärkung seines Staates getroffen hat.

Ich möchte gegen niemanden polemisieren und bitte um Entschuldigung, wenn ich etwas nicht so sage, wie es einige Genossen verstehen - einen zuvor ausgearbeiteten schriftlichen Text habe ich nicht, sondern ich habe mir nur einige Notizen gemacht, nach denen ich auch rede. Ich möchte unsere Position darlegen, wie wir, die Kommunisten der Sowjetunion, und insbesondere ich, einige Fragen verstehen, die von prinzipieller Bedeutung sind.

Da haben die Jugoslawen, vor allem Ranković, 460 in ihren Stellungnahmen, wie Sie sich erinnern werden, erklärt, einige Länder seien angeblich einer auf die Praxis ausgerichteten Einstellung 461 verfallen und wendeten der Entwicklung der Theorie des Marxismus-Leninismus nicht die nötige Aufmerksamkeit zu. Ich denke, dabei haben sie die Sowjetunion im Auge, und ich schließe nicht einmal aus, dass sie mich persönlich meinen. Und falls das so ist, bin ich stolz darauf, dass sie gerade mich so verstehen, und ich bin stolz, dass ich so eine Position beziehe.

Genossen! Als K[arl] Marx und F[riedrich] Engels die Theorie des Klassenkampfes formuliert und entwickelt haben und als Lenin unter veränderten Umständen - in der Periode der imperialistischen Entwicklung des Kapitalismus diese Theorie weiterentwickelt hat, waren die Eroberung der Macht durch die Arbeiterklasse und die Wege zu dieser Eroberung anfänglich nur theoretisch gegeben. Unsere großen Lehrer haben gut genug begründet und dargelegt, wie die Arbeiterklasse und die Werktätigen dem Kapital die Macht entreißen können. Unter der Führung der vom großen Lenin geschaffenen bolschewistischen Partei und unter der direkten Führung Lenins haben die Arbeiterklasse und die Werktätigen Russlands in ihrem Land das Joch der Kapitalisten abgeworfen, die Macht in ihre Hand genommen und die Diktatur des Proletariats errichtet. Nach unserem Land haben auch Ihre Länder entschlossen den Weg der sozialistischen Ent-

459 Die offene Grenze in Berlin erlaubte nicht nur einen Vergleich zwischen den Verhältnissen auf beiden Seiten, sondern gab auch unzufriedenen DDR-Bürger die - vielfach genutzte - Möglichkeit zur Abwanderung in den Westen.

460 Aleksandar Ranković, enger Mitarbeiter Titos, jugoslawischer Innenminister, Chef des Geheimdienstes und der politischen Polizei.

$461 \mathrm{Im}$ russischen Text steht praktizm. Dieser - im Deutschen nicht adäquat wiederzugebende -Ausdruck enthält den Vorwurf an andere Kommunisten, unter Missachtung der marxistisch-leninistischen Doktrin nach rein praktischen Gesichtspunkten zu handeln, die vorgeblich in die Irre führen. 
wicklung beschritten. Für alle unsere Länder sind die Eroberung der Macht durch die Werktätigen und die Errichtung der sozialistischen Ordnung eine Etappe, die hinter uns liegt. Jetzt, Genossen, genügt es schon bereits nicht mehr, vor den Kapitalismus zu treten und die Überlegenheit der Theorie des Marxismus-Leninismus [mit Argumenten] nachzuweisen. In der Periode vor der Revolution hatten wir keine konkreten Möglichkeiten, die Vorteile des Sozialismus gegenüber dem Kapitalismus und die Vorteile der sozialistischen Wirtschaftslenkung praktisch zu demonstrieren. Damals hatten wir nur eine Möglichkeit: die revolutionäre Auseinandersetzung mit dem Kapitalismus zu führen und die Vorteile des Sozialismus gegenüber dem Kapitalismus dadurch nachzuweisen, dass wir sie auf die theoretischen Prinzipien gegründet haben.

Jetzt jedoch, da sich die Staatsmacht in unseren Ländern in den Händen der Arbeiterklasse und des Volkes befindet, da wir sozialistische Staaten errichtet und in der Sowjetunion den Sozialismus aufgebaut haben, stoßen wir praktisch mit der kapitalistischen Welt zusammen und stehen auf allen Feldern der Entwicklung mit ihr in Wettbewerb. Jetzt, Genossen, müssen wir nicht nur die Stärke unserer theoretischen Prinzipien nachweisen, sondern all die materiellen Vorteile darlegen und umfassender enthüllen, die der Sozialismus der Arbeiterklasse und dem gesamten Volk bringt.

Schließlich verläuft in der Sowjetunion schon mehr als 40 Jahre lang die Entwicklung in sozialistischen Bahnen. In diesen Jahren ist eine gewaltige, eine wahrhaft titanische Arbeit geleistet worden, und unser Land hat beim Niveau der Produktion alle Länder der Welt außer den USA eingeholt, und wird jetzt mit Sicherheit auch diese stärkste kapitalistische Macht einholen. Sie wissen, dass wir jetzt nachdrücklich die Frage gestellt haben, die chemische Industrie und besonders die Produktion synthetischer Materialien und der daraus zur Deckung des Bedarfs der Volkswirtschaft und der Versorgung der Bevölkerung produzierten Güter zu beschleunigen. Wenn wir die gestellte Aufgabe erfüllen, und daran haben wir keinen Zweifel, wenn wir jetzt die am meisten fortgeschrittenen kapitalistischen Länder in der Pro-Kopf-Produktion einholen, nimmt dies den kapitalistischen Propagandisten die Möglichkeit, mit Fingern auf uns zu zeigen und zu sagen: Da sieht man, dass die Anhänger des Sozialismus viel über den Sozialismus und über den Wohlstand reden, den die Arbeiterklasse bei der Eroberung der Macht erhält, doch es sind schon 40 Jahre vergangen, seit sie an der Macht sind, und bei ihnen hat der Wohlstand für die Menschen immer noch nicht den Wohlstand erreicht, der vielen Menschen in den hoch entwickelten kapitalistischen Ländern geboten wird.

Ich werde jetzt nicht davon reden, womit wir anfangen mussten und was wir in der Zwischenzeit erreicht haben, als wir den gewaltigen Abstand beseitigt haben, der früher bestand. Jeder Unvoreingenommene, der die Lage der Dinge kennt, weiß, wie dieses Niveau früher war, und wie es jetzt ist. Aber das ist erst der Anfang, und wir schreiten jetzt mit Siebenmeilenstiefeln vorwärts. Die Zeit ist nicht fern, da werden wir die am höchsten entwickelten kapitalistischen Länder in der Pro-Kopf-Produktion nicht nur einholen, sondern auch überholen. Und dann werden alle sagen: Das ist der Sozialismus, das verschafft er der Gesellschaft, den Arbeitenden. 
Daher, Genossen, dürfen wir uns nicht von der Entscheidung über diese Fragen ablenken lassen. Wenn wir jetzt mit dem Kapitalismus ringen, und wir müssen [mit ihm] ringen, weil wir Kommunisten sind, muss dieses Ringen vor allem anderen dazu führen, dass unsere Positionen umfassend gefestigt werden, dass unser Vorposten gestärkt wird. Und so ein Vorposten, wo im Westen die Welt des Sozialismus an die Welt des Kapitalismus angrenzt, ist die DDR.

Der Kampf, Genossen, geht jetzt darum, wer bietet dem Arbeiter, allen Menschen der Arbeit größere Wohltaten. Sehen Sie sich die Meldungen über die Abwanderung der Bevölkerung aus der DDR in den Westen an. Ein sehr großer Teil der Bevölkerung, darunter auch die Jugend, geht aus der DDR nach Westdeutschland: Es gehen viele Arbeiter weg, sogar Parteimitglieder. Sind das denn Feinde der Arbeiterklasse, Feinde des Sozialismus? Nein, Genossen. Das sind Leute, die politisch noch nicht reif sind, welche die Lage im Kapitalismus und im Sozialismus nicht nach einer Analyse der theoretischen Prinzipien beurteilen, sondern nach dem konkreten Vergleich der materiellen Wohltaten: Was hat er in der DDR, und was kann er in Westdeutschland bekommen. So lautet die Frage.

Daher muss man besonders aufmerksam an die Frage der Hilfe für unsere Genossen aus der DDR herangehen, man muss diese Sache real betrachten. Wenn wir die Deutsche Demokratische Republik nicht unterstützen und sie in ihrem Kampf allein lassen, wird sie mit Westdeutschland nicht fertig. Mehr noch, und das dürfen wir nie vergessen, Westdeutschland steht nicht allein da. Wenn man sieht, wie viele Milliarden Mark und Dollar Westdeutschland von den Vereinigten Staaten von Amerika und von anderen kapitalistischen Ländern bekommen hat, oder bedenkt, was der „Marshall-Plan“ war, wird völlig klar, dass dies eine Art internationalistische Hilfe vonseiten der kapitalistischen Kreise der westlichen Länder für die Kapitalisten Westdeutschlands ist. Eine solche Hilfe und Unterstützung gewähren die amerikanischen Kapitalisten auch anderen europäischen Ländern, um den Kapitalisten dieser Ländern zu helfen, ihre Herrschaft in diesen Ländern zu festigen, oder, anders gesagt, den Weg der Bewegung der Arbeiterklasse zu sperren, die Eroberung der Macht durch die Arbeiterklasse und die werktätigen Massen in den Ländern des Kapitals zu verhindern. So und nicht anders lautet die Frage, Genossen. Wir müssen auf Stärke mit Stärke reagieren. Und in diesem Fall meine ich nicht die bewaffnete Stärke. Jetzt die Waffen der einen Länder gegen die anderen einzusetzen, halten wir für falsch.

Folglich, die Frage des Kampfes auf friedlichem, wirtschaftlichem Gebiet, das ist die Frage, wer den Menschen mehr Wohltaten verschafft: die DDR oder Westdeutschland. Um es konkreter zu sagen, in diesen zwei Ländern entscheidet sich durch vergleichende Anschauung die Frage: Wer [besiegt] wen ${ }^{462}$ - Kapitalismus oder Sozialismus? Natürlich lässt sich sagen, wenn man [auf die Dinge] nach den Maßstäben der Geschichte blickt, dass der Klassenkampf dadurch nicht verschwindet und die Theorie des Marxismus-Leninismus sich nicht verändert, dass der Sieg trotzdem letzten Endes auf der Seite der Arbeiterklasse sein wird. Natürlich ist das richtig, doch in diesem Fall geht es nicht um eine weit entfernte

462 Die Frage: Wer wen [besiegt]? galt bei den Kommunisten seit Lenin stets als die zentrale Frage, die alle Überlegungen zu bestimmen hatte. 
Perspektive, sondern um die konkrete Wirklichkeit des heutigen Tages, um das, was schon erreicht und erobert worden ist und was man nicht nur festigen, sondern auch weiterentwickeln muss.

Die erste Position, einige erste Stufen haben die Kräfte des Sozialismus in Deutschland schon erobert. Die 18 Millionen Deutschen, welche die Bevölkerung der DDR bilden, bauen ihren Staat auf sozialistischen Grundlagen auf, sie beschreiten den Weg der sozialistischen Entwicklung zusammen mit allen sozialistischen Ländern und lassen sich dabei von der marxistisch-leninistischen Theorie leiten. Was nun, Genossen, sollen wir etwa den Rückzug antreten? Ich denke, unsere Pflicht ist es gerade, für den Klassenkampf und die Notwendigkeit der internationalen Unterstützung der [Arbeiter-]Klasse nicht nur verbal Verständnis und Anerkennung zu bekunden, sondern auch die erforderliche materielle Unterstützung unseren Klassenbrüdern, unserer gemeinsamen Sache - der Arbeiterklasse, den Werktätigen der DDR - praktisch zu leisten.

Was unsere Delegation betrifft, sind wir bereit, heute den Gürtel etwas enger zu schnallen, um unser gemeinsames sozialistisches Lager zu stärken, den Vorposten des sozialistischen Lagers zu stärken. Und wenn wir durch gemeinsame Anstrengungen unseren deutschen Genossen helfen, den Lebensstandard in der DDR höher zu steigern als in Westdeutschland, wird das eine Anziehungskraft schaffen, die den sozialistischen Einfluss, unsere marxistisch-leninistischen Theorie, noch weiter [als bisher] verbreitet. Dann werden die Menschen die Überlegenheit der marxistisch-leninistischen Theorie erkennen nicht nur durch ihr politisches Bewusstsein, nicht nur durch den Vergleich der in den sozialistischen Ländern verfügbaren politischen Rechte und Bürgerrechte, sondern auch durch den Vergleich der materiellen und kulturellen Wohltaten, die ihnen der Sozialismus gibt, die der Kommunismus mit sich bringt. Sie [an die Zuhörer gewandt] wissen, dass die Fragen des Vorteils des Sozialismus gegenüber dem Kapitalismus vom Volk am leichtesten verstanden werden anhand der Lebensverhältnisse, der materiellen Wohltaten, der politischen Rechte und der Bürgerrechte, welche die sozialistische Ordnung dem Menschen gibt. 463

Man darf nicht außer Betracht lassen, dass die marxistisch-leninistische Theorie anfangs nur einer relativ kleinen Zahl von Arbeitern und dem fortschrittlichen, revolutionären Teil der Intelligenz zugänglich war. Nehmen Sie Russland. Wie viele Kommunisten gab es dort, als die sozialistische Oktoberrevolution stattfand? 80000 Kommunisten, und die Bevölkerung war damals bei über 140 Millionen. Doch dieser kleine Trupp war das geistige Zentrum der Revolution, die, gestützt auf die marxistisch-leninistische Lehre, den Klassenkampf gegen das damals in Russland herrschende gutsherrschaftlich-kapitalistische Joch der Ausbeuterklassen richtete. Dieser Trupp brachte den Willen von vielen Millionen Werktätigen Russlands zum Ausdruck und hatte ihre grenzenlose Unterstützung.

Vielleicht heißt es, Chruschtschow geht zu einfach an die Sache heran. Doch ich möchte so klar und eingängig wie möglich meine Auffassung zum Ausdruck

463 Es folgt der durchgestrichene Satz: Das [die Art, wie das Volk den Vorteil des Sozialismus am leichtesten begreift] sollte man nicht übel nehmen. 
bringen, ich möchte das Wesentliche des Problems hervorheben, damit wir nicht von der Erde abheben, damit wir uns unserer Klasse bewusst sind, sie verstehen, ihre vitalen Interessen richtig verstehen [und] die erforderliche Lösung aller vor uns Kommunisten stehenden Probleme finden.

Wenn ich das sage, polemisiere ich in gewissem Grad gegen die jugoslawischen Politiker, die sich auf grobe Weise von ihrer internationalen Pflicht abgewandt haben und die für uns heiligen Gefühle und Prinzipien der proletarischen Solidarität mit Füßen treten. Das haben sie unlängst besonders anschaulich demonstriert, als wir hier, an diesem Ort, letztes Jahr im November anlässlich des 40. Jahrestages der Oktoberrevolution zusammengekommen sind. Sie haben sich sogar geweigert, sich an der Beratung der Vertreter der kommunistischen und Arbeiterparteien zu beteiligen, sie haben die von dieser Beratung formulierte Deklaration nicht unterschrieben und, nachdem sie das Friedensmanifest unterschrieben hatten, haben sie sich davon auf ihrem BdKJ-Parteitag distanziert. ${ }^{464}$

Genossen, wir, die sowjetischen Kommunisten, verstehen die Lage so, dass es jetzt nicht genügt, von der Überlegenheit unserer revolutionären Theorie zu reden. Die marxistisch-leninistische Theorie gehört nicht nur den Theoretikern, wie es manche Buchgelehrten ein weiteres Mal meinen. Die Arbeiterklasse und das Volk in den sozialistischen Ländern haben unter der Führung ihrer kommunistischen und Arbeiterparteien die Macht erobert und in ihren Ländern die sozialistische Ordnung errichtet. Die Theorie des Marxismus-Leninismus wird jetzt allen Menschen gehören, die sich in ihrem sozialistischen Land am kommunistischen Aufbau beteiligen.

Deswegen würde ich den Genossen Jugoslawen sagen: Sie argumentieren gerne mit der internationalen Pflicht, mit dem Kommunismus und allgemein mit den Kampf gegen den Imperialismus. Aber wie können Sie ernstlich den Imperialismus bekämpfen, wo das Stück Brot, das die Amerikaner Ihnen geben, ${ }^{465}$ Ihnen das Maul stopft? Wie kann der Kapitalismus so eine Kraft ernst nehmen, die gegen ihn „kämpft“ und gleichzeitig von den Almosen der Imperialisten der USA lebt. Denn die Monopolisten der USA können jederzeit an die Adresse der jugoslawischen Politiker sagen: „Wir geben euch keinen Weizen, wenn ihr euch uns gegenüber nicht richtig verhaltet. Dann werdet ihr auf den Knien vor uns rutschen“.

Wir, die sowjetischen Kommunisten, haben nach dem Tod Stalins der praktischen Arbeit viel Aufmerksamkeit gewidmet, den Fragen der wirtschaftlichen Entwicklung unseres Landes und den Fragen der richtigen Anwendung der reichen Möglichkeiten für einen starken Aufschwung aller Wirtschaftszweige des Landes. Und diese Ausrichtung auf die Praxis steht nicht im Gegensatz zur Ent-

464 Die Vertreter des Bundes der Kommunisten Jugoslawiens (BdKJ) hatten nicht an den Beratungen der in den sozialistischen Staaten regierenden kommunistischen Parteien vom 14. bis 16. November 1957 teilgenommen, aber dem Friedensmanifest zugestimmt, das die Vertreter von 68 kommunistischen Parteien aus aller Welt anschließend auf ihrem Treffen vom 16. bis 19. November angenommen hatten. Darin war die Auffasung zum Ausdruck gebracht worden, die vereinte Aktion der „antiimperialistischen Kräfte” könne den Ausbruch von Weltkriegen verhindern.

465 Jugoslawien hatte nicht nur nach dem Bruch Stalins mit Tito 1948 die Wirtschaftsblockade der UdSSR und ihrer Gefolgschaftsstaaten mit amerikanischer materieller Unterstützung überstanden, sondern nahm auch nach der Aussöhnung mit Chruščëv 1955 zu dessen Leidwesen weiter Hilfe aus den USA entgegen. 
wicklung der marxistisch-leninistischen Theorie, sondern begleitet sie vielmehr. Aber sehen Sie selbst: 1948 hatten wir einen Dreijahrplan für die Entwicklung der Viehwirtschaft ausgearbeitet und angenommen. Die drei Jahre vergingen. An eine ganze Reihe von Funktionären wurden damals Orden vergeben (damals hat sich Malenkow mit der Landwirtschaft befasst), doch nichts im Land vermehrte sich, weder Milch noch Fleisch; im Gegenteil, der Viehbestand ist in diesen drei Jahren sogar zurückgegangen.

1955 haben wir einen Sechsjahrplan für die Entwicklung der Viehwirtschaft ausgearbeitet und entsprechende Beschlüsse gefasst. Im Januar sind seitdem drei Jahre vergangen. Und in diesen drei Jahren haben wir diesen Plan zur Erzeugung von Milch und Milchprodukten erfüllt. Widerspricht das etwa unserer Theorie? Würden wir uns hinsetzen und darüber nachsinnen, wie vom theoretischen Standpunkt aus die Entwicklung der Viehwirtschaft zu verstehen sei und was Marx, Engels und Lenin dazu gedacht haben, doch in der Praxis nichts zur erfolgreichen Erfüllung dieses Plans tun, würde uns das nicht weit bringen. Wenn unser Volk im sozialistischen Land keine Milch für die Kinder hätte, kein Fleisch hätte, nicht genug Brot hätte - das wäre eine Verhöhnung der Theorie des Marxismus-Leninismus und nicht ihre Festigung und Weiterentwicklung.

Ich muss viele Reden halten über Mais, Getreide [und] Futtermittel zur Entwicklung der Viehzucht. In manchen Ländern hat man mich kritisiert, ich sei ein Maisfanatiker. ${ }^{466}$ Doch ohne diesen Mais wird es kein Futter für das Vieh geben, folglich wird es keine Milch, kein Fleisch und keine anderen für das Leben der Menschen nötigen Produkte geben, es wird auch keine Kleidung geben. Ohne das gibt es keine Gesellschaft, von der kommunistischen Gesellschaft gar nicht erst zu reden.

Daher läuft die Frage der auf die Praxis ausgerichteten Einstellung auf die Frage hinaus, wie man die Wirtschaft eines sozialistischen Landes unter den gegebenen Umständen am besten so einsetzen kann, dass eine größtmögliche Produktion je aufgewendeter Arbeitseinheit gewährleistet wird, dass die Lebensbedürfnisse des Volkes besser befriedigt werden. Und das lehrt auch die Praxis, das Leben selbst, das lässt sich nur mit einer gekonnten Umsetzung der theoretischen Grundsätze in die Praxis erreichen. Und die Eroberung der Macht durch die Arbeiterklasse, die Werktätigen, der richtige Einsatz der materiellen Produktionsmittel, die in den Dienst des Volkes gestellt werden, und die Sicherung des höchsten Lebensstandards des Volkes in den sozialistischen Ländern - all das wird nur durch eine praktische Tätigkeit der kommunistischen Parteien erreicht, welche die Anstrengungen der Volksmassen in die nötige Richtung auf der Grundlage der marxistisch-leninistischen Lehre lenken und organisieren. Eben das bedeutet die auf die Praxis ausgerichtete Einstellung.

Genossen, als in Sowjetrussland nach der Zerschlagung der Konterrevolution ${ }^{467}$ der Plan der Elektrifizierung aufgestellt wurde, hat Lenin diesen Plan das

${ }^{466} \mathrm{Im}$ Russischen steht kukuruznik, eigentlich = Maisbauer (abgeleitet von kukuruza $=$ Mais). Die hier gewählte Übersetzung soll den mitschwingenden Vorwurf zum Ausdruck bringen, Chruščëv, der den Maisanbau ständig propagierte und mit dem Anspruch eines Experten auftrat, sei darauf fixiert und habe für andere Getreidearten keinen Sinn.

${ }^{467}$ Gemeint ist der Sieg im Bürgerkrieg von 1918 bis 1921. 
zweite Parteiprogramm genannt. Was heißt das? Das heißt, dass er zusammen mit der Theorie zur programmatischen Frage die Verwirklichung des praktischen Plans der Elektrifizierung des Landes gemacht hat, das heißt den Plan zur Hebung der Wirtschaft des Landes, um auf dieser Grundlage die Macht unseres sozialistischen Staates zu festigen, damit wir den Kräften der kapitalistischen Welt widerstehen können, eine stabile Wirtschaft und eine eigene starke Industrie schaffen, eine mechanisierte Großlandwirtschaft haben und den Lebensstandard der Werktätigen spürbar erhöhen. Wie hat sich Lenin denn auf die Praxis ausgerichtet? Ja, er, einer der Begründer des wissenschaftlichen Kommunismus, hat sich mit den Fragen der Praxis, mit den Fragen des Staatsaufbaus, mit den Fragen der Wiederherstellung und Entwicklung der Wirtschaft des Landes befasst. Es steht nicht den Jugoslawen zu, die sich selbst als Leninisten bezeichnen, mit Lenin in Fragen des Theorieverständnisses zu wetteifern. Gerade W[ladimir] I[ljitsch] Lenin war der Ansicht, dass sich die revolutionäre Theorie nicht von der revolutionären Praxis trennen lässt, dass Theorie und Praxis der kommunistischen Partei ein einheitliches Ganzes bilden.

Das, Genossen, sind einige Vorstellungen, die ich zu dieser Frage äußern möchte. Ich habe vielleicht meinem Charakter entsprechend eine gewisse Leidenschaft hineingebracht, vielleicht wäre hier ein professoraler Ton angebracht gewesen. Ich bitte um Entschuldigung. Mir lag daran, grundsätzlich mein Verhältnis zu der Sache zum Ausdruck zu bringen. Es ist natürlich Sache jeder Delegation zu überlegen, wie sie auf den Vorschlag der deutschen Genossen reagiert.

Ich bin von der Delegation der KPdSU und vom Präsidium des ZK bevollmächtigt zu erklären, wie wir die Frage sehen, die von der Delegation der SED aufgeworfen wurde. Wir werden sie unsererseits prüfen und alles, was in unseren Kräften steht, zu tun suchen, um keinen einzigen Schritt zurückzuweichen, sondern nur vorwärts zu gehen. Der Sieg des Sozialismus über den Kapitalismus wird jetzt nicht nur mit der Waffe entschieden, sondern hauptsächlich durch den wirtschaftlichen Wettstreit der zwei Systeme. Dieser wirtschaftliche Wettstreit tritt am deutlichsten in Deutschland zutage. Ich würde so sagen: im Westen Deutschland, im Mittelosten, in Albanien. Ich rede nicht von Volkschina. China ist so ein Koloss, der mit seiner Revolution die ganze kapitalistische Welt erschüttert hat und weiter erschüttert mit seinen Erfolgen. Doch Albanien ist ein kleines Land. Es ist das sozialistische Vorbild für die muslimische arabische Welt, was große Bedeutung hat. Deutschland, das ist Westeuropa, das ist die westliche Welt. Wir müssen von unserer Seite alles tun, um die eroberten Positionen zu festigen und nicht zurückzuweichen. Und die Genossen, die an vorderster Front kämpfen, müssen in jeder Weise unterstützt und keinem willkürlichen Geschick überlassen werden.

Das ist alles, was ich zu dieser Frage sagen wollte.

Korrigiertes Stenogramm

Übersetzt aus dem russischen Originaltext RGANI, fond 52, opis` 1 , delo 443, Bl. 146-160 